\title{
Theory of orthogonal flow in elastic chambers and bio-bags
}

\author{
K. Kosorin \\ Slovak Academy of Sciences, Bratislava, Slovakia
}

\begin{abstract}
This study presents a theory of the orthogonal flow of Newtonian fluid in an elastic chamber. The elastic chamber is a space bounded by a movable material boundary $\bar{S}$ that has a single orifice and is pliable with respect to normal forces. The characteristic feature of this flow is that the fluid motion is perpendicular to moving $\bar{S}$ and $\bar{S}$ to the one-parameter level surfaces $S_{i}$ inside the chamber. The orthogonal flow domain is confined to such parts of the chamber interior $V$, for which the level function $\Phi$ exists defining the system of level surfaces $S_{i}$, by means of the equation $\Phi(x, y, z)=\varphi_{i}$ with the set of constant parameters $\varphi_{i}$, $i=1,2 \ldots, \infty$. This flow property enables us to define the three-component velocity field $\mathbf{a}(u, v, w)$ by the single vector $\boldsymbol{q}$ normal to $S_{i}$ for whole $\bar{V}$. By means of vector algebra and the Navier-Stokes description of the shear stress tensor, a mathematical formulation of the mass and momentum conservation laws for orthogonal flow has been derived as the main result of the theory. The certainty and the uncertainty of the direction field of flow were used as the determining factors in this process. A constitutive form of the level function $\Phi(x, y, z)$ as the tool for obtaining the system of level surfaces $S_{i}$ for chambers of real geometry has been proposed and applied for the sphere and slanted ellipsoid chambers.

The study of liquor dynamics effects on hydrocephalus development in the system of brain ventricles inspired this work. It is assumed that the orthogonal flow theory can effectively simplify the mathematical description of a certain kind of fluid flow in elastic chambers as well as in some cavities and bags of live organisms.
\end{abstract}

Key words: single-orifice elastic chamber, orthogonal flow, directional cosines, directional field of flow, level function, level surface system, stream lines, intrinsic derivatives, constitutive form of level function, Navier-Stokes equations. 


\section{Introduction}

\subsection{Brain disorder motivation}

The orthogonal flow in an elastic chamber is understood as fluid motion perpendicular to moving wall of the chamber. Such flow or flow approaching its properties can occur in certain circumstances in elastic chambers of artificial origin, e.g., balloons, balls, and bubbles. In bio-world, it can be in some kinds of octopus and jellyfish with reactive motion as well as fluid bags and cavities in biologic tissues. The motivation to work on the presented theory arises from the latter case. It began during a hydrodynamic study of some hydrocephalic phenomena in connection with the cerebrospinal fluid (CSF) flow in the brain ventricle system (Kosorin [1]). Just two lateral ventricles, K1 as left and K2 as right chamber behave like the elastic single-orifice chambers.

\subsection{Oscillation of the cerebrospinal fluid (CSF) in the brain ventricle system as the single-orifice chamber flow}

The endoscopy technique (Novak et al. [2]) has allowed direct monitoring of inner swinging movement of a filament neoplasm at the connecting orifice, the "foramina of Monro" between brain chambers K1, K2 and third K3. Kosorin's comparative calculation has shown that the liquor dynamics in the chamber system is preferably characterized by oscillating liquor through the foramina Monro orifices $S_{0}$, connecting the third chamber K3 with the first two chambers, $\mathrm{K} 1$ and K2. It was approximately identified with the liquor velocity $v=2.0\left[\mathrm{~m} . \mathrm{s}^{-1}\right]$ and the pulsating mass flow rate ca $2.0\left[\mathrm{~cm}^{3} \mathrm{~s}^{-h}\right]$. This flow regime seems to be laminar because its Reynolds number for the orifice diameter between 0.5 and $1.0 \mathrm{~cm}$ is less than 200. But the total liquor (CSF) secretion and the effluence from the brain chambers system into liquor routes are cca one-third of $\mathrm{ml} / \mathrm{min}$, Nadvornik [3]. It is however more than 360 times less than the oscillating discharge between K1, K2 and K3. Therefore, the oscillating CSF flow between chambers is a significant property of the brain ventricular system dynamics.

Kurtcuoglu et al. [4] presents the numerical simulation of 3D oscillating flow of CSF in the third ventricle (chamber K3 in Fig. 1). The 2D numerical model of pulsatile movement of CSF in the sagittal plane of K3 has been treated by Cheng and Bilston [5]. Simulation of the oscillating CSF flow in the interior of lateral ventricles $\mathrm{K} 1$ and $\mathrm{K} 2$ does not appear in these studies. The phenomenon has been very complex and behaves like the object of the presented theory. Therefore a new methodical approach can be useful here. The orthogonal flow theory would be a step in that direction.

\section{The orthogonal flow hypothesis}

\subsection{Definition and physical description of the orthogonal fluid flow in the single-orifice elastic chambers}

This study deliberates on the describing of real fluid motion in a space domain $\bar{V}$ confined by an elastic, flexible, and moving wall having an orifice. Such an 
elastic chamber of volume $\bar{V}$ is assumed to exist as a fluid cavity in pliable environment $V_{e}$; see Fig. 1. This theory considers (specific) dynamic state of the elastic system $\bar{V}+V_{e}$, producing in $\bar{V}$ the fluid motion in which a fluid moves always in the direction to or from the boundary (or orifice) without any streams tangential to the chamber wall. The orthogonal flow is considered to exist in the whole 3D domain $\bar{V}>0$ confined by the elastic material boundary $\bar{S}$ and the passable, orifice surface $S_{0}$. Then, the whole chamber boundary $S_{c}$ is given by

$$
S_{c}=\bar{S}+S_{0} \text {. }
$$

The chamber orifice $S_{0}>0$ anchors the chamber in space. Its form and position are given as constants in time. The closed curve $k_{0}$ confines the surfaces $\overline{\boldsymbol{S}}, S_{0}$, and all $S_{i}$ as their common space line. The shape of $V$ and $V_{i}$ is an oval segment with the common single edge.

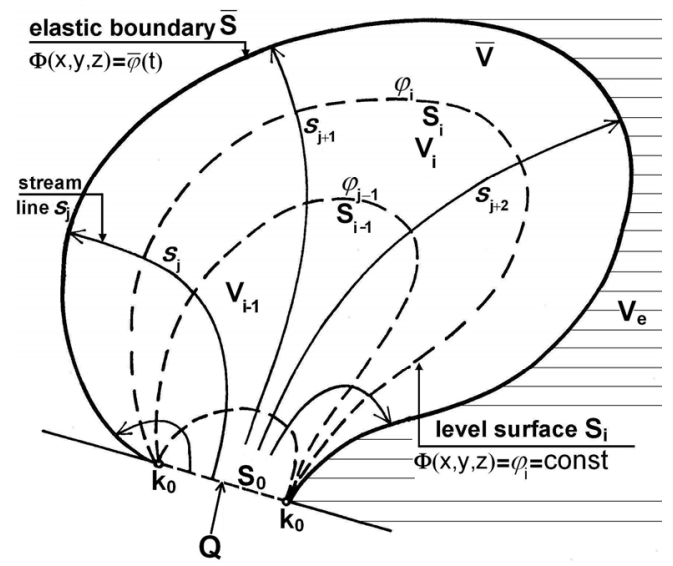

Figure 1: $\quad$ Scheme of the orthogonal system $\left(S_{i}, S_{j}\right)$ for elastic chamber $\bar{V}$ in pliable environment $V_{e}$ with movable interface $\bar{S}$. The oneparameter level surfaces $S_{i}$ follows from the level function $\Phi(\mathrm{x}, \mathrm{y}, \mathrm{z})$ $=\varphi_{i}=$ const.

\subsection{Directional field of flow. Orthogonal system $\left(S_{i}, s_{j}\right)$ of the level surfaces $S_{i}$ and the stream lines $s_{j}$}

The orthogonal flow theory originates from the idea of existence of the directional field of flow. Then one has to define an object toward which the velocity vectors $a(x, t)$ are perpendicular. Beginning at $\bar{S}$ and proceeding in conformity with the idea, one intuitively fills the space $\bar{V}$ with a system of surfaces $S_{i}, i=0,1,2 \ldots i c$ and a system of space curves $s_{j}, j=1,2 \ldots j c$ normal to $S_{i}$, so that they meet the basic property of a coordinate system $\left(S_{i}, s_{j}\right)$. This property allows each point $(x, y, z)$ of the domain $V$ to be uniquely determined by the crossing point of the single surface $S_{i}$ and the single curve $s_{j}$, i.e., by the number pair $(i, j)$. Regular surfaces $S_{i}$ do not have any common points, except the orifice 
curve $k_{0}$, in which all $S_{i}$ cross each other and $S_{0}$ with $\bar{S}$ confines $\bar{V}$. Space curves $s_{j}$ are assumed to be identical with the stream line system of flow.

The idea of the orthogonal flow requires to state the direction field of flow through the $S_{i}$ system, permitting to define and formulate the orthogonal velocity field $\boldsymbol{q}(\boldsymbol{x} t)$. The first and decisive step is anticipating the surfaces $S_{i}$ to be the socalled level surfaces determined within the chamber domain $\bar{S}$ by means of the one-parameter relation

$$
\Phi(x, y, z)=\varphi_{i}, \quad \mathrm{i}=1,2 \ldots
$$

in which $\varphi_{i}$ parameters for individual $S_{i}$ are constant. The level function $\Phi$ in (2.2.1) determines the direction cosine field $\left({ }^{1} c,{ }^{2} c,{ }^{3} c\right)$ of the outside normal to $S_{i}$ at each point on $S_{i}$, by means of the partial derivatives of $\Phi$. Varying $\varphi_{i}$ in (2.2.1), one ills the domain $\bar{V}$ with surfaces $S_{i}$. Therefore, the level function $\Phi$ alone defines the field of direction cosines $\left({ }^{1} c,{ }^{2} c,{ }^{3} c\right)$ for the entire $\bar{V}$. Then, the unit normal vector $\boldsymbol{n}$ of $S_{i}$ is defined by

$$
\boldsymbol{n}={ }^{1} c \mathbf{i}+{ }^{2} c \mathbf{j}+{ }^{3} c \mathbf{k}
$$

and its divergence by

$$
\operatorname{div} \boldsymbol{n}={ }^{1} \mathrm{c}_{\mathrm{x}}+{ }^{2} \mathrm{c}_{\mathrm{y}}+{ }^{3} \mathrm{c}_{\mathrm{z}}
$$

see Korn and Korn [6], x,y,z mark the partial derivatives. There the unit vectors $(\boldsymbol{i}, \boldsymbol{j}, \boldsymbol{k})$ are the base vectors (constant) and the direction cosine field is a function of position. As the vector $\boldsymbol{n}$ creates the field of tangents to $s_{j}$, one is able to build the responding system $s_{j}$ using the direction field defined by (2.2.1). This field, of course, has to be identical with the direction field of the flow investigated. Moreover, because the field of the unit vector $\boldsymbol{n}$ has been uniquely defined by $\left({ }^{1} c,{ }^{2} c,{ }^{3} c\right)$, the direction field of the orthogonal flow is uniquely determined by $\boldsymbol{n}$. Finally, its stream line system $s_{j}$ can be built by means of the characteristic system of equations if written for continuity equation (3.0.3) in the next chapter.

Because (2.2.1) describes also the moving boundary $\bar{S}$, its mobility requires the parameter $\varphi_{i}$ in (2.2.1) to become a continual function of time $\varphi_{i}=\bar{\varphi}(t)$. Therefore, treating with $\bar{S}$, a unique function of time $\bar{\varphi}(t)$ has to be applied on the right-hand side of (2.2.1) instead of the constant $\varphi_{i}$. Then

$$
\Phi(x, y, z)=\bar{\varphi}(t)
$$

describes the moving boundary $\bar{S}$. From (2.2.4) it follows that one of the three independent variables $\mathrm{x}, \mathrm{y}, \mathrm{z}$ in $\Phi(x, y, z)$ becomes a function of the remaining two and time $t$. It applies for any moving boundary problem concerning the elastic chamber.

\subsection{The constituent form of the level function $\Phi$ for a single-orifice chamber}

The one-parameter level surface system (2.2.1) is the crucial feature of the theory. The first property is the condition that the level function $\Phi$ in $(2.2 .1)$ 
yields such a system $\left(S_{i}, S_{j}\right)$, which is orthogonal. The second one follows from the singular behavior of the direction field of flow upon the closed curve $k_{o}$. It means that all $S_{i}$ 's intersect upon $k_{o}$. Due to this singularity, the orifice curve $k_{o}$ becomes the determinative element of the level function $\Phi$. These properties can be expected at $\Phi$, which is written in the particular constitutive form

$$
\Phi=\frac{\Phi_{N}}{\Phi_{D}}+\Phi_{M}
$$

This form is able to produce the $\mathrm{S}_{\mathrm{i}}$ system (2.2.1) for a large range of chamber shapes if the functions $\Phi_{N}, \Phi_{D}, \Phi_{M}$ are chosen properly. The functions $\Phi_{N}$ and $\Phi_{D}$, set in the ratio in (2.3.1), provide the singular behavior of $\Phi$ along $k_{o}$, if they satisfy the conditions

$$
\Phi_{N}(x, y, z)=0 ; \Phi_{D}(x, y, z)=0 \text { on } k_{o}
$$

In such a case, equations (2.3.2) define uniquely the closed curve $k_{o}$ confining the chamber orifice. The function $\Phi_{M}(x, y, z)$ in (2.3.1) remains arbitrary because of the necessary ability of the constitutive form (2.3.1) to create the level functions $\Phi$ for chambers of optional shapes. $\Phi_{M}$ gains such an ability if written as the sum

$$
\Phi_{M}=\sum a_{m} \Phi_{m} ; m=1,2, \ldots M
$$

in which the functions $\Phi_{m}(x, y, z)$, the real coefficients $a_{m}$, and their total number $M$ are free. Due to their characteristics, $\Phi_{N}$ and $\Phi_{D}$ can be named the orifice functions and $\Phi_{M}$ the shaping function.

Constructing the one-parameter level surface system $S_{i}$ by means of the constitutive form (2.3.1) is possible for chambers, the geometry of which yields the unique field of direction cosines through partial derivatives of $\Phi$. Therefore, the knowledge of the level function $\Phi(x, y, z)$ for a given chamber is a decisive step in utilizing the theory.

\subsection{The orthogonal velocity field}

Generally, in every chamber volume $V$ a fluid may move in any direction or path. Then, the velocity vector $\boldsymbol{a}=\boldsymbol{i} u+\boldsymbol{j} v+\boldsymbol{k} w$ yields its components $a_{n}$ normal to $S_{i}$ through the direction cosines by the well-known relation

$$
a_{n}={ }^{1} c u+{ }^{2} c v+{ }^{3} c w
$$

Decomposition of any vector $\boldsymbol{a}$ into its normal component $\boldsymbol{a}_{n}$ and other two components $\boldsymbol{a}_{1}$ and $\boldsymbol{a}_{2}$ satisfies the vector sum

$$
\boldsymbol{a}=a_{1} \boldsymbol{n}_{1}+a_{2} \boldsymbol{n}_{2}+a_{n} \boldsymbol{n}
$$

in which $\boldsymbol{n}_{1}, \boldsymbol{n}_{2}$ and $\boldsymbol{n}$ are unit vectors of its components differing generally from zero. One may be however interested in a special type of the velocity field in $\bar{V}$ - i.e., for which the following applies: 


$$
a_{1}=0 ; \quad a_{2}=0 ; a_{n}=q
$$

The velocity vector $q=n q$ of such flow should be in each point of domain $\bar{V}$ perpendicular to any $S_{i}$ because of (2.2.2). Therefore, the system of space curves $s_{j}$, being perpendicular to the $S_{i}$ surfaces $(2.2 .1)$, has to be identical with the stream line system of such flow, which can be named as orthogonal. The system of the one-parameter level surfaces (2.2.1) thereby became the system of the normal cross-flow surfaces. The 3-D velocity field of such flow has been defined by the normal component $q$ and by the direction cosine field. Indeed, projecting $q$ on coordinate axes, one obtains all three velocity components through the relations

$$
u={ }^{1} c q ; \quad v={ }^{2} c q ; \quad w={ }^{3} c q
$$

These equations together with (2.3.3) show the main feature of the presented theory. It describes the 3-D velocity field by means of its normal component $q$ only. Such a step means significant simplification of the flow image in $\bar{V}$.

\section{Mass conservation law for orthogonal flow}

After using (2.4.4), the continuity equation as the differential equivalent of the mass conservation law for a compressible fluid flow takes the form

$$
\frac{\partial \rho}{\partial t}+\frac{\partial(\rho q)}{\partial s}+\rho q \operatorname{div} \boldsymbol{n}=0
$$

For an incompressible fluid, this equation simplifies into

$$
\frac{\partial q}{\partial s}+q \operatorname{div} \boldsymbol{n}=0
$$

The partial derivatives of the specific mass $\rho$ and the normal velocity $q$ with respect to stream line $s$ signify the intrinsic derivatives along $s$. Writing the equation (3.0.2) in the explicit (not intrinsic) form one obtains

$$
{ }^{1} c \frac{\partial q}{\partial x}+{ }^{2} c \frac{\partial q}{\partial y}+{ }^{3} c \frac{\partial q}{\partial z}=-q \operatorname{div} \boldsymbol{n}
$$

If the unit vector $\boldsymbol{n}(x, y, z)$ is known, equation (3.0.3) represents a partial differential equation of the first order for a single unknown variable $q(x, y, z, t)$. Its corresponding characteristic system of equations defines the stream line system $s_{j}$.

\section{Momentum conservation law for orthogonal flow}

In performing the task stated, we proceed from the known integral form of the momentum conservation law, see Lojcianskij [7], 


$$
\int_{v} \rho\left(\frac{d \mathbf{a}}{d t}-\mathbf{F}\right)=\int_{S} \mathbf{p}_{n} d S
$$

in which the vector

$$
\mathbf{p}_{n}=\mathbf{n} \Pi={ }^{1} c \mathbf{p}_{1}+{ }^{2} c \mathbf{p}_{2}+{ }^{3} c \mathbf{p}_{3}
$$

of forces acting up to fluid volume $V$ through its boundary surface $S$ is defined by stress tensor $\Pi$ and unit vector $\mathbf{n}$ of the normal to $S$, where the vectors $\mathbf{p}_{1}, \mathbf{p}_{2}, \mathbf{p}_{3}$ are given through a linear combination of the stress tensor components $p_{i j}$ by Navier-Stokes (N-S).

\subsection{Certainty and uncertainty of direction field of flow as the determining factor of the differential form of momentum conservation law}

Equation (4.0.1) in 3D space corresponds with three independent equations for three velocity vectors, $\boldsymbol{u}=u \boldsymbol{i}, \boldsymbol{v}=v \boldsymbol{j}, \boldsymbol{w}=w \boldsymbol{k}$. In the orthogonal flow theory presented, this three-unknown-vector field has been defined by means of a single vector $\boldsymbol{q}=\boldsymbol{n} q$, where $\boldsymbol{n}=\boldsymbol{n}(x, y, z)$ is a directional unit vector of the external normal to level surfaces (2.2.1). Because the vectors $\boldsymbol{u}, \boldsymbol{v}, \boldsymbol{w}$ ceased to be mutually independent, consistence of the theory requires transformation of the law (4.0.1) into a single momentum equation only as a crucial point of the theory.

With the exception of the last but decisive step, we would apply the known procedures used by deriving the N-S equations. Transforming the law (4.0.1) into its differential equivalent, the first step is made by means of the Gauss integral theorem. Applying this theorem upon the N-S tensor components, one substitutes the surface integral in (4.0.1) by the volume integral according to equation

$$
\int_{S} \mathbf{p}_{n} d S=\int_{V}\left[\mathbf{i}\left(-p_{x}+\mu \Delta u\right)+\mathbf{j}\left(-p_{y}+\mu \Delta v\right)+\mathbf{k}\left(-p_{z}+\mu \Delta w\right)\right] d V
$$

where the symbol $\Delta$ indicates the Laplace operator on velocity field and $\mu$ is the viscosity coefficient.. This relation enables to express the integral law of momentum conservation (4.0.1) through the volume integral of vector

$$
\int_{V}\left(\mathbf{i} A_{1}+\mathbf{j} A_{2}+\mathbf{k} A_{3}\right) d V=0
$$

with the scalar components $\left(A_{1}, A_{2}, A_{3}\right)$ given by

$$
\begin{aligned}
& A_{1}=\frac{d u}{d t}+\frac{1}{\rho} p_{x}+\Omega_{x}-v\left(\Delta u+\frac{1}{3}(\operatorname{div} \mathbf{a})_{x}\right) \\
& A_{2}=\frac{d v}{d t}+\frac{1}{\rho} p_{y}+\Omega_{y}-v\left(\Delta v+\frac{1}{3}(\operatorname{div} \mathbf{a})_{y}\right)
\end{aligned}
$$




$$
A_{3}=\frac{d w}{d t}+\frac{1}{\rho} p_{z}+\Omega_{z}-v\left(\Delta w+\frac{1}{3}(\operatorname{div} \mathbf{a})_{z}\right)
$$

Here appear kinematic viscosity $v$, pressure $p$ and external forces potential $\Omega$. The spatial partial derivatives are expressed through indexes x,y,z. The standard proceeding based on arbitrariness of the volume $V$ transforms (4.1.2) in to

$$
\mathbf{i} A_{1}+\mathbf{j} A_{2}+\mathbf{k} A_{3}=0
$$

The next step in current obtaining the N-S equations is projecting the vector $\boldsymbol{A}$ $=\boldsymbol{i} A_{1}+\boldsymbol{j} A_{2}+\boldsymbol{k} A_{3}$ in (4.1.6) successively in all three directions $x, y, z$. It yields the $\mathrm{N}$ $S$ equations as the scalar solution of (4.1.6) defined by zero components

$$
A_{1}=0, A_{2}=0, A_{3}=0,
$$

The vector equation (4.1.6) is general and valid also for the orthogonal flow. Therefore, the method of scalar solutions of (4.1.6) will be decisive in the following treatment. The way used gives just the N-S equations (4.1.7). For obtaining the other particular solution of (4.1.6), it was important to perceive and utilize that the vector projection into a selected direction with a scalar result is equivalent to the scalar product of two vectors. For example, the N-S equations (4.1.7) have appeared as the projections of $\boldsymbol{A}$ into coordinate axes. But the scalar products $\boldsymbol{i} \boldsymbol{A}, \boldsymbol{j} \boldsymbol{A}$, and $\boldsymbol{k} \boldsymbol{A}$ produce the same result, what is important for further steps.

The aim is however to obtain just a single scalar equation from the vector one (4.1.6). One can get it by projecting the vector $\boldsymbol{A}$ into the direction of the unit vector of the normal $\mathrm{n}$ defined by equation (2.2.2). Because such a projection is equivalent to the scalar product $\boldsymbol{n} \boldsymbol{A}$, multiplying (4.1.6) by $\boldsymbol{n}$ from (2.2.2) and respecting the scalar products $\boldsymbol{i} \boldsymbol{i}=\boldsymbol{j} \boldsymbol{j}=\boldsymbol{k} \boldsymbol{k}=\mathbf{1}, \boldsymbol{i} \boldsymbol{j}=\boldsymbol{i} \boldsymbol{k}=\boldsymbol{j} \boldsymbol{k}=\mathbf{0}$ one obtains the wanted scalar solution of (4.1.6) in the form

$$
{ }^{1} c A_{1}+{ }^{2} c A_{2}+{ }^{3} c A_{3}=0
$$

With components (4.1.3)-(4.1.5), equation (4.1.8) becomes an equivalence of the N-S equations (4.1.7) for the normal velocity component $q$ and the pressure p. After the continuity equation (3.0.1), it yields the second equation for two dependent variables. It means the closed system of equations for the orthogonal flow has been just obtained. Of course, in case of the compressible fluid, a suitable function $\rho(p)$ or $p(\rho)$ is assumed to exist for this purpose. Nevertheless, appearance of (4.1.8) demands still certain explanation.

The way of obtaining the equation (4.1.8) has been correct and without any limitation regarding the direction field $\boldsymbol{n}$. Therefore, (4.1.8) also applies to general, unspecified fluid flow. But the N-S system (4.1.7) does the same. This plight is, of course, solvable by the fact that the N-S system (4.1.7) can be derived from equation (4.1.8) as well as. Therefore, these two scalar solutions of equation (4.1.6) are not mutually independent and solution (4.1.8) is superior. 


\subsection{Momentum equations of the orthogonal flow of viscous fluids}

Deriving the resulting explicit form of the momentum equation (4.1.8) is now only a routine question. It consists mostly of elimination procedures. For the velocity component elimination in the system of (4.1.3) to (4.1.5), equation (2.3.4) is prepared. The formal algebraic properties of intrinsic derivatives and direction cosines (their sum of squares $=1$, see Korn and Korn [6]) will be utilized there. Then the summation of the intrinsic total derivatives of the velocity components in (4.1.8) seems to be the simplifying step for transformation

$$
{ }^{1} c \frac{d u}{d t}+{ }^{2} c \frac{d v}{d t}+{ }^{3} c \frac{d w}{d t} \Rightarrow \frac{d q}{d t}
$$

Equation (4.2.1) applies generally, not just for the steady direction field $\boldsymbol{n}$ derived from the level surfaces (2.2.1). Specifying now $d q / d t$ according to the same properties of direction cosines, one obtains

$$
\frac{d q}{d t}=\frac{\partial q}{\partial t}+q \frac{\partial q}{\partial s}
$$

Summation of the pressure and external volume force gradients enable one to use the intrinsic operator $\partial / \partial s$. Then, the momentum equation (4.1.8) can be written down in the semiexplicit form

$$
\frac{\partial q}{\partial t}+\frac{\partial}{\partial s}\left(\frac{q^{2}}{2}+\Omega\right)+\frac{1}{\rho} \frac{\partial p}{\partial s}=v S_{f}
$$

where the right-hand side $v S_{f}$ represents the shear stress tensor by N-S. Therefore, individual members representing friction forces in (4.1.8) make the sum

$$
S_{f}={ }^{1} c\left(\Delta u+\frac{1}{3} \frac{\partial d i v \mathbf{a}}{\partial \mathbf{x}}\right)+{ }^{2} c\left(\Delta v+\frac{1}{3} \frac{\partial d i v \mathbf{a}}{\partial y}\right)+{ }^{3} c\left(\Delta w+\frac{1}{3} \frac{\partial d i v \mathbf{a}}{\partial z}\right)
$$

Use of the intrinsic formalism $\partial / \partial$ simplifies this sum, yielding

$$
S_{f}={ }^{1} c \Delta u+{ }^{2} c \Delta v+{ }^{3} c \Delta w+\frac{1}{3} \frac{\partial d i v \mathbf{a}}{\partial s}
$$

In summing these Laplacians, the sum of squares of direction cosines $(=1)$ appears and simplifies the treatment. Then after eliminating the velocity vector $\mathbf{a}$ through (2.4.4), the sum $S_{f}$ becomes

$$
S_{f}=\Delta q+q \sum_{i=1}^{3} i_{c} \Delta^{i} c+\frac{1}{3} \frac{\partial}{\partial s}\left(\frac{\partial q}{\partial s}+q \operatorname{div} \mathbf{n}\right)
$$


Therefore applying (4.2.4), one can write down the momentum equation (4.1.8) in the semi-explicit form

$$
\frac{\partial q}{\partial t}+\frac{\partial}{\partial s}\left(\frac{q^{2}}{2}+\Omega\right)+\frac{1}{\rho} \frac{\partial p}{\partial s}=v\left(\Delta q+\sigma q+\frac{1}{3} \frac{\partial}{\partial s}\left(\frac{\partial q}{\partial s}+q \operatorname{div} \mathbf{n}\right)\right)
$$

where the coefficient $\sigma(x, y, z)$ has been given by

$$
\sigma(x, y, z)=\sum_{i=1}^{3}{ }^{i} c \Delta^{i} c={ }^{1} c \Delta^{1} c+{ }^{2} c \Delta^{2} c+{ }^{3} c \Delta^{3} c
$$

This equation is an equivalence of the N-S equations for the orthogonal flow of the Newtonian fluid. Together with the continuity equation (3.1.1), they form the system of partial differential equations for the pressure $p$ and the velocity $q$. This system is just closed, if the direction field $\boldsymbol{n}$ of the vector $\boldsymbol{q}$ is known.

The fully explicit form of the momentum equation (4.2.5) means to write it without the intrinsic formalism $\partial / \partial s$. But the use of all three spatial derivatives should require a sensible longer record of (4.2.5). Moreover, the conservation law (4.2.5) for the incompressible fluid can be put down very shortly, i.e.

$$
\frac{\partial q}{\partial t}+\frac{\partial E}{\partial s}=v S_{f}
$$

where

$$
E=\frac{1}{2} q^{2}+\frac{p}{\rho}+\Omega
$$

is the unit energy of flow. The right-hand side $v S_{f}$ in (4.2.11) defines the slope of the unit energy line, i.e., the energy loses along the stream line s with

$$
S_{f}=\Delta q+\sigma q
$$

The momentum equation (4.2.5) as well as its conservation form (4.2.11) has been derived for the orthogonal flow for which the direction field $\boldsymbol{n}$ follows from the level surface system (2.2.1).

\section{Some applications for chambers of simple geometry}

\subsection{Application of the constitutive equation for sphere and slanted ellipsoid}

Obtaining the level function $\Phi$ for a spherical chamber with a single orifice through the constituent relations (2.3.1)-(2.3.3) is very simple. The choice of an orifice and its boundary $k_{0}$ is a starting step. If the circular orifice is set at the plane $z=0$, then two spherical surfaces are suitable to consider for conditions (2.3.2). For the numerator in (2.3.1), it may be

$$
\Phi_{N} \equiv x^{2}+y^{2}+z^{2}-r_{0}^{2}
$$


where $r_{0}$ is the orifice radius. The denominator demands other sphere, but crossing the first one through $k_{0}$. Because its radius $r$ is arbitrary, it is most simple to choose that $r \rightarrow \propto$. Then,

$$
\Phi_{D} \equiv z
$$

The building of $\Phi$ ends at the choice $\Phi_{M} \equiv 0$. Applying these results in (2.3.1) and (2.2.1), one can write equation of the level surface system $S_{i}$

$$
\left(x^{2}+y^{2}+z^{2}-r_{0}^{2}\right) / z=2 z_{i}
$$

where the variable parameter $\varphi_{i} \Rightarrow z_{i}$ in this case means the position (altitude) of central points of the spherical surfaces $S_{i}$ above the plane $z=0$.

An example of application with nonzero $\Phi_{M}$ is still relatively simple even for the slanted ellipsoidal chamber; see Fig.2. If the chamber is symmetrical with respect to plane $y=0$, then three points on the original (starting) $\bar{S}=\overline{S_{3}}$ are needed to put down the sufficient conditions to get the level function $\Phi$ uniquely. While the orifice functions can stay given by (5.1.1), (5.1.2), the shaping $\Phi_{M}$ requires $M=2$. Its simplest form is $\Phi_{M}=a_{1} x+a_{2} z$ and its use in (2.3.1) and (2.2.1) yields the level surface system

$$
\left(x^{2}+y^{2}+z^{2}-r_{0}^{2}\right) / z+a_{1} x+a_{2} z=\varphi_{i}
$$

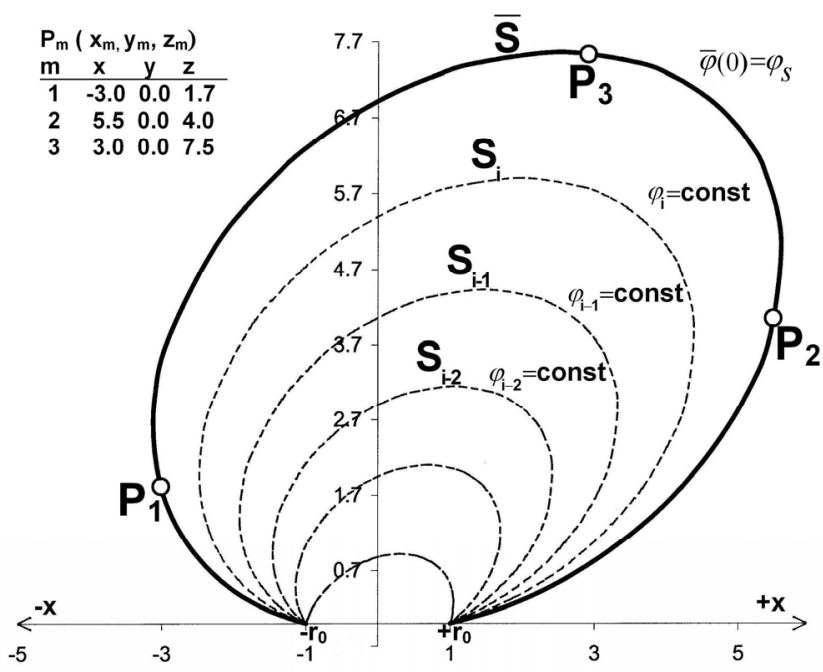

Figure 2: $\quad$ Level surface systems $S_{i}$ for the slanted ellipsoid chamber. Its level function (5.1.4) originates from the constitutive form (2.3.1) with $a_{l}=0.333$ and $a_{2}=-0.146$ after applying $\Phi_{\mathrm{m}}$ at the points $\mathrm{P}_{\mathrm{m}}$, $m=1,2,3$. 
Besides $a_{1}$ and $a_{2}$, a original value of $\varphi_{i}$ is also unknown here. But three linear equations for these parameters follow from (6.1.4), if written down for three suitable points $(x, 0, y)$ on $S_{3}$. Of course, the orifice functions alone have fixed two other (singular) points $x_{1,2}= \pm r_{0}$ on $S_{3}$.. The choice of points $(x, 0, z)$ and the obtained numerical values of $a_{1}, a_{2}$, and $\varphi_{3}$ are seen in Fig.2. The resulting chamber is an inclined ellipsoid. Its crossing with the planes $x=$ const. and $y=$ const. creates ellipses, while $z=$ const. makes circles.

\subsection{Parameters divn and $\sigma=\sum{ }^{i} c \Delta^{i} c$ for governing equations of the orthogonal flow in the spherical chambers}

Presence of the vector $\left({ }^{1} c,{ }^{2} c,{ }^{3} c\right)$ in both basic equations demonstrates impact of the chamber geometry on velocity and pressure fields inside the chamber. Therefore, it is interesting to know what values the respective parameters may reach. In the event of a spherical chamber, equation (5.1.3) allows us to answer this question. Components of the directional vector $\boldsymbol{n}$ resulting from (5.1.3) are

$$
{ }^{1} c=2 x z / \rho_{S} ;{ }^{2} c=2 y z / \rho_{S} ;{ }^{3} c=\left(z^{2}-x^{2}-y^{2}+r_{0}^{2}\right) / \rho_{S}
$$

and the denominator

$$
\rho_{S}=x^{2}+y^{2}+z^{2}+r_{0}^{2} \text {. }
$$

According to (2.2.3), the scalar components (5.2.1) grant the divergence

$$
\operatorname{div} \boldsymbol{n}={ }^{1} c_{x}+{ }^{2} c_{y}+{ }^{3} c_{z}=4 z / \rho_{S}
$$

One can determine the value of the sum $\sigma$ for the direction field (5.2.1) by applying (4.2.6). It is possible even without deriving Laplace's $\Delta^{i} c$ with the result

$$
\sigma=\sum_{i=1}^{3}-{ }^{i} c \Delta^{i} c=-\frac{4 z^{2}}{\rho_{s}^{2}}
$$

where $\rho_{s}$ is the same as in (5.2.2).

\section{Summary}

The study presents the theory of orthogonal flow of a real fluid in elastic chambers with a single orifice. The elastic chamber is understood to be a space bounded by movable material boundary $\bar{S}$, which is pliable with respect to normal forces. The nonslip condition and the continuity of flow on its material boundary are those physical factors that lead to the existence hypothesis for the orthogonal flow. The characteristic feature of this flow is that the fluid motion is perpendicular to the moving wall $\bar{S}$ and to one-parameter level surfaces $S_{i}$ 
defined in $\bar{V}$ by the level function $\Phi$ in the equation $\Phi(x, y, z)=\varphi_{i}$ through the constant values of the parameters $\varphi_{i,} i=1,2 \ldots \infty$.

Using the vector algebra tools, the continuity equation (3.0.1) and the momentum equation (4.2.5) for compressible fluids and their equivalents (3.0.2), (4.2.11) for incompressible fluids have been derived as the main product of the theory. The obtained result as the closed system of two equations for the pressure $p(x, y, z, t)$ and the normal velocity $q(x, y, z, t)$ represents the mass and momentum conservation laws for the real Newtonian fluids with the shear stress tensor by Navier-Stokes. The presented theory carries the possibility to simulate the dynamics of 3-D orthogonal flow in a single-orifice elastic chamber using the system of two equations only.

For obtaining the one-parameter level surface system $S_{i}$ for any chambers of real geometry, the constitutive form (2.3.1) of the level function $\Phi(x, y, z)$ has been proposed and applied for the sphere and ellipsoid chamber as a sample.

The flows near or equal to the orthogonal flow are assumed to occur in certain technical objects as well as in some cavities, bags and cells of live organisms. The orthogonal flow should be regarded as a special kind of flow.

\section{References}

[1] Kosorin, K.; The pressure and flow regime in brain liquor space; Final research report; APVV project no SK - CZ-022006; Bratislava, March 2006

[2] Novak, Z., Chrastina, J., Riha, I., Krupa, P., Kajan, J.; Endoscopic surgeries of the ventricular system - presurgical planning, image transmission and virtual reality. Proc. of 18th Intern. Congress and Exhibition CARS 2004

[3] Nadvornik, P.; Contemporary neurosurgery; VEDA, SAV, 1983; (in Slovak)

[4] Kurtcuoglu, Vartan, Soellinger, M., Summers, P., Boomsma, K., Poulikakos. D., Boesinger, P., Ventikos, Y.; Computational investigation of subject- specific cerebrospinal fluid flow in the third ventricle and aqueduct of Sylvius; Journal of Biomech., Volume 40, Issue 6, Pages 1235-1245, 2007.

[5] Cheng, S. \& Bilston, L.; A fluid structure flow interaction model of the ventricular system. Journal of Biomechanics, Volume 39, Supplement 1, Page S367, Abstracts of the $5^{\text {th }}$ World Congress of Biomechanics, 2006

[6] Korn,G.A.-Korn,T.M., Math. handbook for scientist and engineers; NY 1968

[7] Lojcianskij, L.G., Mechanics of liquids and gases, I. II., SNTL, Prague, 1954 